\title{
Produtividade, eficiência de uso da água e qualidade tecnológica de cana-de-açúcar submetida a diferentes regimes hídricos
}

\author{
Emídio Cantídio Almeida de Oliveira( ${ }^{(1)}$, Fernando José Freire ${ }^{(2)}$, Alexandre Campelo de Oliveira ${ }^{(2)}$, \\ Djalma Euzébio Simões Neto(3), Alexandre Tavares da Rocha ${ }^{(1)}$ e Laércio Alves de Carvalho ${ }^{(4)}$
}

\begin{abstract}
(1)Universidade Federal Rural de Pernambuco (UFRPE), Unidade Acadêmica de Serra Talhada, Fazenda Saco, s/no, Caixa Postal 063, CEP 56903-070 Serra Talhada, PE. E-mail: emidio@uast.ufrpe.br, alexandre@uast.ufrpe.br (2)UFRPE, Departamento de Agronomia, Rua Dom Manoel de Medeiros, s/nº, Dois Irmãos, CEP 52171-900 Recife, PE. E-mail: f.freire@depa.ufrpe.br, alexandre_solos@hotmail.com (3)UFRPE, Estação Experimental de Cana-de-açúcar de Carpina, Rua Ângela Cristina C.P. de Luna, s/non, Novo, CEP 55810-700 Carpina, PE. E-mail: desn@oi.com.br (4)Universidade Estadual de Mato Grosso do Sul, Unidade Universitária de Dourados, Cidade Universitária de Dourados, Zona Rural, CEP 79800-000 Dourados, MS. E-mail: Icarvalh@uems.br
\end{abstract}

Resumo - O objetivo deste trabalho foi avaliar a produtividade de colmos e de açúcar, a eficiência de uso da água e os atributos tecnológicos de cinco variedades de cana-de-açúcar de maturação precoce e seis de maturação média à tardia, submetidas ao regime de sequeiro $(1.141,4 \mathrm{~mm})$ e à irrigação plena $(1.396,6 \mathrm{~mm})$. Utilizou-se o delineamento experimental de blocos ao acaso, com quatro repetições. A produtividade de colmos das variedades RB92579, RB72454 e SP81-3250 apresentou ganhos superiores a 180\%, com uso da irrigação. A irrigação promoveu aumentos na produtividade de açúcar superiores a $200 \%$, nas variedades RB92579 e RB943365. A irrigação plena proporcionou maior eficiência de uso da água, com produção média de $70,2 \mathrm{~kg} \mathrm{ha}^{-1} \mathrm{~mm}^{-1}$ de colmos a mais em comparação ao regime de sequeiro. Com exceção das variedades RB72454, RB763710 e RB943365, não houve diferença nos atributos tecnológicos entre a irrigação plena e o regime de sequeiro. As variedades RB92579 e SP81-3250 de maturação média à tardia se destacam quanto à produtividade de colmos e de açúcar e à eficiência de uso da água, e são recomendadas para estudos de resposta à irrigação pela cana-de-açúcar.

Termos para indexação: Saccharum, cana-planta, irrigação plena, sequeiro.

\section{Productivity, water use efficiency, and technological quality of sugarcane subjected to differents water regimes}

\begin{abstract}
The objective of this work was to assess stalk and sugar yield, water use efficiency, and technological attributes of five early maturing and six medium to late maturing sugarcane varieties with no irrigation $(1,141.4 \mathrm{~mm})$ and under full irrigation $(1,396.6 \mathrm{~mm})$. A randomized complete block design, with four replicates, was used. Stalk yield for varieties RB92579, RB72454, and SP81-3250 showed gains above $180 \%$ with irrigation. Irrigation increased sugar yield in more than $200 \%$ in the RB92579 and RB943365 varieties. Full irrigation provided greater water use efficiency, producing, in average, $70.2 \mathrm{~kg} \mathrm{ha}^{-1} \mathrm{~mm}^{-1} \mathrm{more}$ stalks than under no irrigation. Except for the varieties RB72454, RB763710, and RB9433665, there were no differences regarding technological attributes between full irrigation and no irrigation. The medium to late maturing varieties RB92579 and SP81-3250 have higher stalk and sugar yield and better water use efficiency, and are recommended for studies on sugarcane response to irrigation.
\end{abstract}

Index terms: Saccharum, cane plant, full irrigation, no irrigation.

\section{Introdução}

A expansão do setor sucroalcooleiro tem aumentado o cultivo da cana-de-açúcar em solos ácidos, de baixa fertilidade natural e com teores elevados de alumínio tóxico, bem como em regiões com precipitações pluviais irregulares e inferiores à necessidade da cultura, o que inibe o crescimento radicular e a absorção de água (Zheng \&Yang, 2005; Efeoglu et al., 2009) e limita o aumento da produtividade dos canaviais brasileiros.
Para o uso eficiente da água pela cana-de-açúcar, é fundamental identificar a necessidade hídrica responsável pelas máximas produções (Wiedenfeld \& Enciso, 2008). O consumo anual de água pela cana-de-açúcar varia de 1.500 a 2.500 mm (Doorenbos $\&$ Kassam, 1994). No entanto, a distribuição irregular e a redução dos volumes de chuvas durante o crescimento vegetativo da cultura causam prejuízos, com queda na produção e mortalidade das soqueiras, o que força a renovaçãoprecoce docanavial(Dantas Netoetal.,2006). 
O crescimento da cana-de-açúcar, caracterizado por quatro fases fenológicas (germinação, perfilhamento, crescimento elevado e maturação) (Gascho, 1985) pode apresentar maior ou menor impacto na produtividade agrícola, conforme se acentua o deficit hídrico entre os estádios de crescimento (Inman-Bamber \& Smith, 2005).

O efeito do estresse hídrico nas diferentes fases de desenvolvimento da cana-de-açúcar ainda não é bem elucidado na literatura (Wiedenfeld, 2000). Contudo, sabe-se que o grau de injúria promovido pelo estresse depende consideravelmente do estádio fenológico da planta e da duração do estresse (Farias et al., 2008a). Períodos prolongados com baixa disponibilidade hídrica afetam negativamente a qualidade tecnológica da cultura e a produtividade de colmos e de açúcar (Inman-Bamber, 2004). De acordo com Machado et al. (2009), a disponibilidade hídrica adequada reduz significativamente as perdas e proporciona condições favoráveis para o desenvolvimento vegetativo da planta, quando o estresse hídrico é mais acentuado nas fases iniciais de crescimento. A maior suscetibilidade da cana-de-açúcar ao estresse hídrico na fase de crescimento elevado promove efeitos mais severos na redução das taxas de crescimento do colmo, na produção de fitomassa e no rendimento de sacarose (Robertson et al., 1999; Silva \& Costa, 2004), o que confirma os resultados obtidos por Wiendenfeld (1995), ao verificar perdas de produtividade com a redução da disponibilidade hídrica na fase de elongação do colmo.

Os investimentos na tecnologia da irrigação e o lançamento de novas variedades são algumas das alternativas de avanço tecnológico para os canaviais brasileiros (Raizer \& Vencovsky, 1999; Farias et al., 2008a). A combinação da irrigação com variedades edafoclimaticamente adaptadas proporciona incrementos na qualidade do caldo e na produção de colmos e de açúcar (Carvalho et al., 2009). Entretanto, grande parte dos canaviais irrigados estão sendo conduzidos em áreas inadequadas, de baixa fertilidade, com uso de variedades não responsivas à irrigação, o que limita o crescimento vertical da cultura e eleva o custo dessa tecnologia.

A produção de açúcar e de álcool pela cana-de-açúcar irrigada depende de diversos fatores, como a quantidade de água aplicada pela irrigação, a variedade utilizada e o tipo de solo e de clima da região (Dantas Neto et al.,
2006). Oliveira et al. (2009) verificaram incrementos na produtividade de colmos com irrigação, enquanto Souza et al. (1999) constataram diferentes respostas das variedades a esse manejo. Segundo Inman-Bamber \& Smith (2005), a água é considerada fator limitante à produção da cana-de-açúcar, pois, à medida que sua disponibilidade aumenta, a cultura expressa seu potencial produtivo com diferentes respostas entre as variedades.

O objetivo deste trabalho foi avaliar a produtividade de colmos e de açúcar, a eficiência de uso da água e os atributos tecnológicos de cinco variedades de cana-de-açúcar de maturação precoce e seis de maturação média à tardia submetidas ao regime de sequeiro e à irrigação plena.

\section{Material e Métodos}

Os experimentos foram conduzidos em campo, na área agrícola da Estação Experimental de Cana-de-açúcar de Carpina, da Universidade Federal Rural de Pernambuco, no Município de Carpina, PE (7051'13"S, 35²1 '10"W, a $180 \mathrm{~m}$ de altitude), entre julho de 2006 e novembro de 2007, no ciclo de cana-planta. O clima da região, conforme Köppen, é do tipo Ams, tropical chuvoso de monção, com verão seco.

O solo da área experimental é classificado como Argissolo Amarelo distrófico abrupto (Santos et al., 2006), de textura arenosa e argilosa. A caracterização física e química do solo foi realizada em amostras coletadas nas camadas de $0-20,21-40$ e $41-60 \mathrm{~cm}$ (Tabela 1).

O preparo do solo consistiu de gradagem para destruição dos restos culturais, previamente dessecados com herbicida (glifosato) e incorporação do calcário, seguida da abertura dos sulcos de plantio. Para correção do solo, utilizou-se calcário na dose de $465 \mathrm{~kg} \mathrm{ha}^{-1}$, calculado pelo método da neutralização do $\mathrm{Al}$ trocável ou da elevação dos teores de $\mathrm{Ca}$ e $\mathrm{Mg}$ trocáveis, tendose considerado 3,0 $\mathrm{cmol}_{\mathrm{c}} \mathrm{dm}^{-3}$ como nível crítico de $\mathrm{Ca}+\mathrm{Mg}$. Na adubação de plantio, foram aplicados no fundo do sulco $30 \mathrm{~kg} \mathrm{ha}^{-1}$ de $\mathrm{N}, 120 \mathrm{~kg} \mathrm{ha}^{-1}$ de $\mathrm{P}_{2} \mathrm{O}_{5} \mathrm{e}$ $70 \mathrm{~kg} \mathrm{ha}^{-1}$ de $\mathrm{K}_{2} \mathrm{O}$. Aos 90 dias após o plantio (DAP), foi feita adubação de cobertura, tendo-se aplicado $50 \mathrm{~kg} \mathrm{ha}^{-1}$ de $\mathrm{N}$ e $50 \mathrm{~kg} \mathrm{ha}^{-1}$ de $\mathrm{K}_{2} \mathrm{O}$ nas linhas da cana, seguida de incorporação manual.

Dois experimentos foram conduzidos separadamente: um sob irrigação plena $(1.396,6 \mathrm{~mm})$ e outro 
em regime de sequeiro (1.141,4 $\mathrm{mm})$. Em ambos os experimentos, utilizou-se o delineamento de blocos ao acaso, com quatro repetições. Foram avaliadas 11 variedades de cana-de-açúcar: cinco variedades de maturação precoce (SP79-1011, RB813804, RB863129, RB872552 e RB943365) e seis de maturação média a tardia (RB72454, RB763710, SP78-4764, SP81-3250, RB867515 e RB92579), perfazendo 88 parcelas experimentais. As parcelas foram constituídas de cinco sulcos de cana-de-açúcar, com espaçamento de $1,10 \mathrm{~m}$ e $10 \mathrm{~m}$ de comprimento. A área útil da parcela abrangeu os três sulcos centrais, com $8 \mathrm{~m}$ de comprimento.

O plantio foi realizado manualmente. Os colmossemente foram repartidos, tendo-se mantido três gemas por rebolo, e distribuídos nos sulcos de plantio, para atingir 18 gemas por metro. No experimento de sequeiro, o plantio foi realizado em julho de 2006 e a colheita em novembro de 2007, o que totalizou 17 meses de crescimento. No experimento irrigado, o plantio foi realizado em outubro de 2006 e a colheita em outubro de 2007, com 12 meses de crescimento.

A precipitação efetiva, obtida com auxílio de pluviômetro (MI-028, Sonda Terra, Piracicaba, SP), instalado na área experimental, foi calculada após a dedução das perdas de água excedentes da precipitação total recebida, por percolação abaixo dos $0,6 \mathrm{~m}$ de profundidade, em ambos os regimes hídricos avaliados. O uso total de água (UTA) pelas variedades foi calculado a partir da precipitação efetiva, do esgotamento da umidade do perfil e da irrigação (campo irrigado) (Singh et al., 2007). A eficiência de uso da água (EUA) foi calculada de acordo com a equação:

EUA $\left(\mathrm{kg} \mathrm{ha}^{-1} \mathrm{~mm}^{-1}\right)=$ produtividade de colmos $\left(\mathrm{Mg} \mathrm{ha}^{-1} \times 10^{-3} / \mathrm{UTA}(\mathrm{mm})\right.$.

A irrigação foi manejada de forma plena, tendose utilizado a lâmina de água necessária para suprir a soma diária da evapotranspiração da cultura (ETc) que correspondesse a $50 \%$ da água disponível para a profundidade de $0,4 \mathrm{~m}$, nos três primeiros meses, e de 0,6 $\mathrm{m}$ até os 300 DAP (Tabela 1). Para tanto, foram considerados os resultados da capacidade de campo e do ponto de murcha permanente do solo até as respectivas profundidades, bem como a precipitação e a eficiência $(75 \%)$ do sistema de irrigação utilizado. Para a irrigação, utilizou-se o sistema de aspersão convencional do tipo canhão móvel, com bocal de quatro polegadas de diâmetro e vazão de $54 \mathrm{~m}^{3} \mathrm{~h}^{-1}$ sob pressão de $40 \mathrm{~m}$ de coluna de água, disposto em

Tabela 1. Caracterização química e física do solo da área experimental, de 2006 a 2007.

\begin{tabular}{|c|c|c|c|}
\hline \multirow[t]{2}{*}{ Parâmetro } & \multicolumn{3}{|c|}{ Profundidade $(\mathrm{cm})$} \\
\hline & $0-20$ & $21-40$ & $41-60$ \\
\hline & \multicolumn{3}{|c|}{ Parâmetros químicos } \\
\hline pH $\mathrm{H}_{2} \mathrm{O}$ & 5,4 & 5,2 & 5,0 \\
\hline $\mathrm{P}_{\text {Mehlich }}\left(\mathrm{mg} \mathrm{dm} \mathrm{dm}^{-3}\right)$ & 8 & 7 & 6 \\
\hline $\mathrm{H}+\mathrm{Al}\left(\mathrm{cmol}_{\mathrm{c}} \mathrm{dm}^{-3}\right)$ & 6,4 & 6,8 & 6,8 \\
\hline $\mathrm{Al}\left(\mathrm{cmol}_{\mathrm{c}} \mathrm{dm}^{-3}\right)$ & 0,2 & 0,5 & 0,7 \\
\hline $\mathrm{Ca}\left(\mathrm{cmol}_{\mathrm{c}} \mathrm{dm}^{-3}\right)$ & 1,6 & 1,0 & 0,75 \\
\hline $\operatorname{Mg}\left(\mathrm{cmol}_{\mathrm{c}} \mathrm{dm}^{-3}\right)$ & 1,2 & 0,5 & 0,5 \\
\hline $\mathrm{K}\left(\mathrm{cmol}_{\mathrm{c}} \mathrm{dm}^{-3}\right)$ & 0,07 & 0,05 & 0,03 \\
\hline CTC $\left(\mathrm{cmol}_{\mathrm{c}} \mathrm{dm}^{-3}\right)$ & 9,33 & 8,50 & 8,20 \\
\hline V $(\%)$ & 31,4 & 20,0 & 17,0 \\
\hline \multirow[t]{2}{*}{$\mathrm{m}(\%)$} & 6,3 & 22,7 & 26,1 \\
\hline & \multicolumn{3}{|c|}{ Parâmetros físicos ${ }^{(1)}$} \\
\hline Densidade $\left(\mathrm{kg} \mathrm{m}^{-3}\right)$ & 1,44 & 1,36 & 1,39 \\
\hline Areia $\left(\mathrm{g} \mathrm{kg}^{-1}\right)$ & 769,4 & 689,4 & 661,0 \\
\hline Silte $\left(\mathrm{g} \mathrm{kg}^{-1}\right)$ & 61,4 & 46,4 & 54,8 \\
\hline Argila $\left(\mathrm{g} \mathrm{kg}^{-1}\right)$ & 169,2 & 264,2 & 284,2 \\
\hline$\Theta_{\mathrm{cc}}\left(\mathrm{m}^{3} \mathrm{~m}^{-3}\right)$ & 0,15 & 0,21 & 0,21 \\
\hline$\Theta_{p m p}\left(m^{3} m^{-3}\right)$ & 0,083 & 0,110 & 0,140 \\
\hline LU (mm) & 7,4 & 9,7 & 7,3 \\
\hline Textura & Franco arenosa & Franco-argilo-arenosa & Franco-argilo-arenosa \\
\hline
\end{tabular}

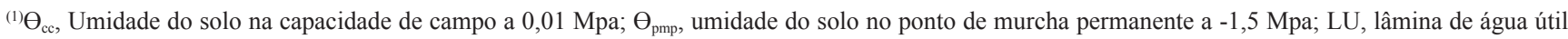
calculada a partir de $50 \%$ da lâmina total. 
quatro posições de espera, para realizar a sobreposição completa. Nos 60 dias que antecederam a colheita, não foi adicionada água por irrigação, para reduzir o crescimento vegetativo e estimular a maturação fisiológica da cana. A precipitação pluvial para este período foi de $96 \mathrm{~mm}$ (Figura 1).

A ETc $(\mathrm{mm})$ foi calculada pela equação: $\mathrm{ETc}=\mathrm{ECA} \times \mathrm{Kp} \times \mathrm{Kc}$, em que ECA é a evaporação do tanque Classe A (mm); Kp é o coeficiente do tanque Classe A; e Kc é o coeficiente de cultura. Os valores de Kp foram obtidos a partir dos dados de velocidade do vento, umidade relativa e evaporação do tanque classe $\mathrm{A}$, instalado próximo à área experimental com vegetação rasteira contendo bordadura de $10 \mathrm{~m}$ (Doorenbos \& Pruitt, 1976). Para o Kc, seguiram-se os estádios de desenvolvimento da cana-de-açúcar descritos em Oliveira et al. (2010).

Os colmos da área útil de cada parcela experimental foram pesados, ainda no campo e com auxílio de dinamômetro PR30-3000, com precisão de 500 g, (Líder, Araçatuba, SP), para determinar a produtividade de colmos $\left(\mathrm{Mg} \mathrm{ha}{ }^{-1}\right)$. Após a pesagem, realizouse amostragem aleatória de dez colmos, que foram encaminhados ao Laboratório Agroindustrial da Usina Petribu S/A, em Pernambuco. As amostras foram passadas em forrageira, e foram coletadas subamostras
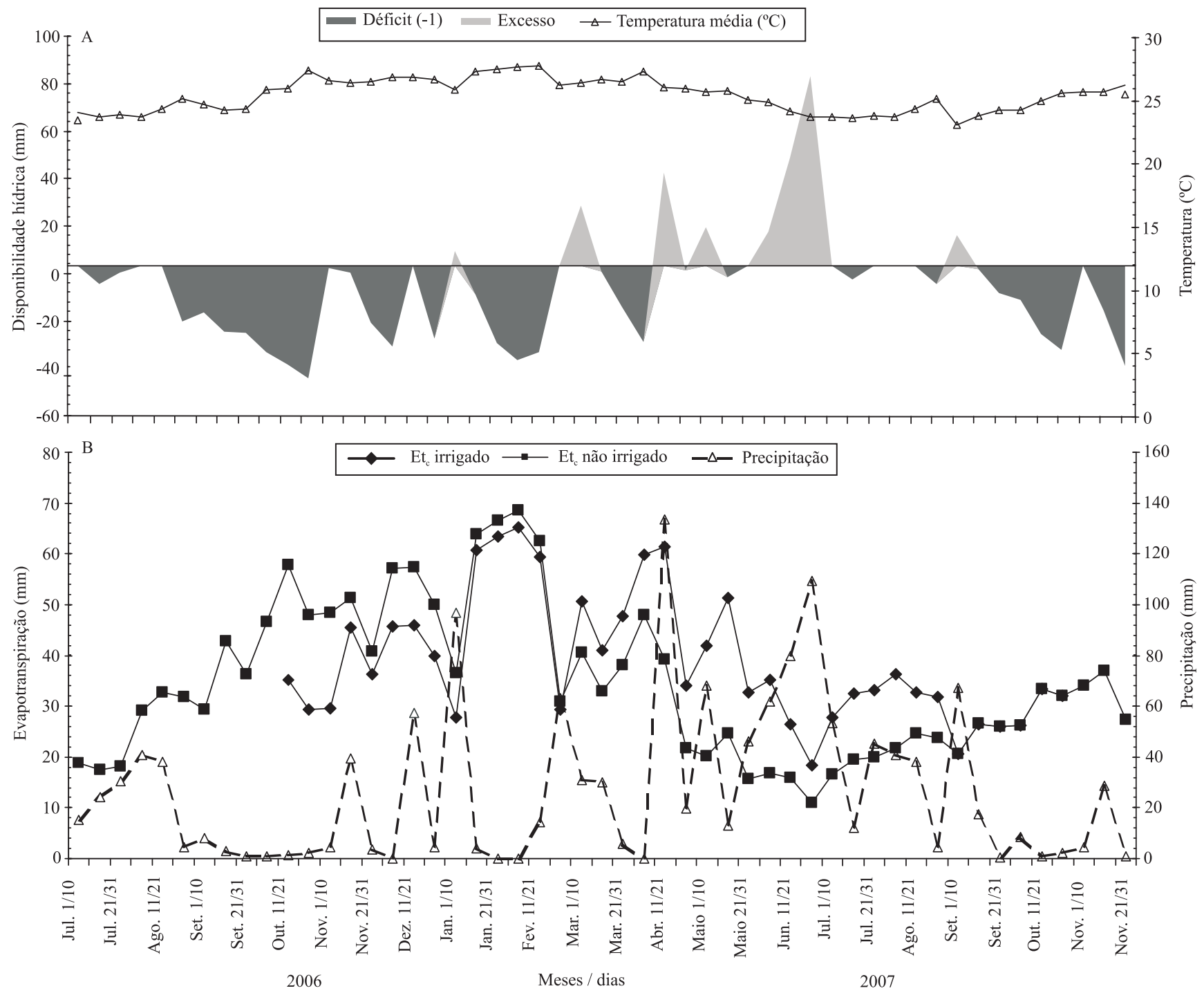

Figura 1. Balanço e regime hídrico observados nos experimentos de variedades de cana-de-açúcar cultivadas em regime hídrico de sequeiro e sob irrigação plena. A, disponibilidade hídrica; B, evapotranspiração da cultura. 
para determinação dos atributos tecnológicos da cana-de-açúcar, conforme metodologia descrita por Fernandes (2003). As subamostras foram submetidas à prensa hidráulica automática MA098, (Marconi Equipamentos para Laboratório, Ltda., Piracicaba, $\mathrm{SP}$ ), sob pressão de $250 \mathrm{~kg} \mathrm{~cm}^{-2}$, durante $1 \mathrm{~min}$, tendo-se coletado o caldo do colmo. No caldo, foram quantificados os sólidos solúveis totais ( ${ }^{\circ}$ Brix) com refratômetro digital Autopol 589, (Tecnal Equipamentos para Laboratórios, Piracicaba, SP), dotado de correção automática de temperatura. A percentagem de sacarose aparente no caldo (Pol) foi obtida por meio de sacarímetro automático Autopol 589, (Tecnal, Equipamentos para Laboratórios, Piracicaba, SP). Foram determinados a percentagem de fibra, o açúcar teórico recuperável e a percentagem de sacarose no colmo (PC), de acordo com as equações descritas em Fernandes (2003) e Carvalho et al. (2008). A produtividade de açúcar $\left(\mathrm{Mg} \mathrm{ha}^{-1}\right)$ foi quantificada pelo produto da $\mathrm{PC}$ com a produtividade de colmos.

Os dados obtidos em ambos os experimentos foram submetidos à análise conjunta de variância em grupos de experimentos e delineamento de blocos ao acaso. Os dados qualitativos de produtividade de colmo e de açúcar, EUA e atributos tecnológicos foram comparados pelo teste de Scott-Knott, a 5\% de probabilidade, com uso do programa SAS (SAS Institute, 1999).

\section{Resultados e Discussão}

No regime de sequeiro, a precipitação efetiva e a temperatura média entre julho de 2006 e novembro de 2007 foram de $1.141,4 \mathrm{~mm}$ e $24,7^{\circ} \mathrm{C}$, respectivamente. No experimento irrigado, de outubro de 2006 a outubro de 2007 , a temperatura média foi de $24,9^{\circ} \mathrm{C}$ e a precipitação efetiva de $926,6 \mathrm{~mm}$, tendo-se adicionado por irrigação $470 \mathrm{~mm}$ de água, o que totalizou 1.396,6 mm de água disponível.

A produtividade de colmos apresentou diferença significativa entre os regimes hídricos avaliados, com ganhos médios de $116 \mathrm{Mg} \mathrm{ha}^{-1}$ sob irrigação plena (Tabela 2). As variedades RB92579, RB72454 e SP81-3250 obtiveram ganhos acima de 180\%, enquanto as variedades RB863129, RB867515 e SP79-1011 tiveram os menores incrementos com uso da irrigação. Nas demais variedades, a irrigação proporcionou incremento médio de $143 \%$.

Carvalho et al. (2009), em estudo com lâminas de irrigação, em Argissolo, também relataram incrementos para os ciclos de cana-planta e cana-soca, menores que os observados no presente trabalho. Entretanto,

Tabela 2. Produtividade de colmos e de açúcar, eficiência de uso da água e respectivos incrementos em diferentes variedades de cana-de-açúcar cultivadas em regime hídrico de sequeiro e sob irrigação plena, de 2006 a $2007^{(1)}$.

\begin{tabular}{|c|c|c|c|c|c|c|c|c|c|c|c|c|}
\hline \multirow[t]{2}{*}{ Variedade } & \multicolumn{4}{|c|}{ Produtividade de colmos $\left(\mathrm{Mg} \mathrm{ha}^{-1}\right)$} & \multicolumn{4}{|c|}{ Produtividade de açúcar $\left(\mathrm{Mg} \mathrm{ha}^{-1}\right)$} & \multicolumn{4}{|c|}{ Eficiência de uso da água $\left(\mathrm{kg} \mathrm{ha}^{-1} \mathrm{~mm}^{-1}\right)$} \\
\hline & Sequeiro & Irrigado & Incremento & Incremento $(\%)$ & Sequeiro & Irrigado & Incremento & Incremento $(\%)$ & Sequeiro & Irrigado & Incremen & emento $(\%)$ \\
\hline & \multicolumn{12}{|c|}{ Variedades de maturação precoce } \\
\hline SP79-1011 & $71,2 \mathrm{a}$ & $155,0 \mathrm{c}$ & 83,8 & 118 & $10,7 \mathrm{a}$ & $25,6 \mathrm{~d}$ & 14,9 & 139 & $62,4 \mathrm{a}$ & $111,0 \mathrm{c}$ & 48,6 & 78 \\
\hline RB813804 & $79,9 a$ & $177,5 \mathrm{c}$ & 97,6 & 122 & $13,9 \mathrm{a}$ & $28,4 \mathrm{~d}$ & 14,5 & 104 & $70,0 \mathrm{a}$ & $127,0 \mathrm{c}$ & 57,0 & 81 \\
\hline RB863129 & $87,8 \mathrm{a}$ & $181,5 \mathrm{c}$ & 93,7 & 107 & $13,2 \mathrm{a}$ & $28,8 \mathrm{~d}$ & 15,6 & 118 & $76,9 a$ & $129,9 \mathrm{c}$ & 53,0 & 69 \\
\hline RB872552 & $76,7 \mathrm{a}$ & $192,5 \mathrm{c}$ & 115,8 & 151 & $12,2 \mathrm{a}$ & $31,5 \mathrm{c}$ & 19,3 & 158 & $67,2 \mathrm{a}$ & $137,8 \mathrm{c}$ & 70,6 & 105 \\
\hline \multirow[t]{2}{*}{ RB943365 } & $80,6 a$ & $193,2 \mathrm{c}$ & 112,6 & 140 & $10,5 \mathrm{a}$ & $33,4 \mathrm{c}$ & 22,9 & 218 & $70,6 \mathrm{a}$ & $138,3 \mathrm{c}$ & 63,7 & 90 \\
\hline & \multicolumn{12}{|c|}{ Variedades de maturação média à tardia } \\
\hline RB72454 & $68,0 \mathrm{a}$ & $191,6 \mathrm{c}$ & 123,6 & 182 & $11,0 \mathrm{a}$ & $24,2 \mathrm{~d}$ & 13,2 & 120 & $59,6 \mathrm{a}$ & $137,2 \mathrm{c}$ & 77,6 & 130 \\
\hline RB763710 & $79,2 \mathrm{a}$ & $211,9 b$ & 132,7 & 168 & $11,7 \mathrm{a}$ & $27,9 \mathrm{~d}$ & 16,2 & 138 & $69,3 \mathrm{a}$ & $151,7 \mathrm{~b}$ & 82,4 & 119 \\
\hline SP78-4764 & $76,2 \mathrm{a}$ & $179,2 \mathrm{c}$ & 103,0 & 135 & $11,8 \mathrm{a}$ & $28,6 \mathrm{~d}$ & 16,8 & 142 & $66,7 \mathrm{a}$ & $128,3 \mathrm{c}$ & 61,6 & 92 \\
\hline SP81-3250 & $83,1 \mathrm{a}$ & $232,5 b$ & 149,4 & 180 & $12,8 \mathrm{a}$ & $37,3 b$ & 24,5 & 191 & $72,8 \mathrm{a}$ & $166,5 b$ & 93,7 & 129 \\
\hline RB867515 & $87,6 a$ & $186,0 \mathrm{c}$ & 98,4 & 112 & $13,7 \mathrm{a}$ & $29,8 \mathrm{c}$ & 16,1 & 117 & $76,8 \mathrm{a}$ & $133,2 \mathrm{c}$ & 56,4 & 73 \\
\hline RB92579 & $90,1 \mathrm{a}$ & $255,6 \mathrm{a}$ & 165,5 & 184 & $13,6 \mathrm{a}$ & $42,6 \mathrm{a}$ & 29,0 & 213 & $78,9 \mathrm{a}$ & $183,0 \mathrm{a}$ & 104,1 & 132 \\
\hline Média & $80,0 \mathrm{~B}$ & $196,0 \mathrm{~A}$ & 116,0 & 145 & $12,3 \mathrm{~B}$ & $30,7 \mathrm{~A}$ & 18,4 & 151 & $70,1 \mathrm{~B}$ & $140,3 \mathrm{~A}$ & 69,8 & 99 \\
\hline $\mathrm{CV}(\%)$ & \multicolumn{4}{|c|}{13,54} & \multicolumn{4}{|c|}{15,51} & \multicolumn{4}{|c|}{12,88} \\
\hline Variedade (Var) & \multicolumn{4}{|c|}{$5,87 * *$} & \multicolumn{4}{|c|}{$6,32 * *$} & \multicolumn{4}{|c|}{$6,10 * *$} \\
\hline Regime hídrico (Rh) & \multicolumn{4}{|c|}{$847,10 * *$} & \multicolumn{4}{|c|}{$670,00 * *$} & \multicolumn{4}{|c|}{$590,48 * *$} \\
\hline Var x Rh & \multicolumn{4}{|c|}{$3,59 * *$} & \multicolumn{4}{|c|}{$4,37 * *$} & \multicolumn{4}{|c|}{$3,38 * *$} \\
\hline
\end{tabular}

${ }^{(1)}$ Médias seguidas de letras iguais, maiúsculas nas linhas e minúsculas nas colunas, não diferem entre si pelo teste de Scott-Knott, a 5 \% de probabilidade. **Significativo pelo teste $\mathrm{F}$, a $1 \%$ de probabilidade. 
quando as lâminas de irrigação foram inferiores à água armazenada no solo, os ganhos na produção não foram significativos, o que corrobora os resultados obtidos por Wiedenfeld \& Enciso (2008), que, ao avaliar diferentes regimes de irrigação na região semiárida do Texas, EUA, não verificaram ganhos na produção de colmos com lâminas de irrigação inferiores à capacidade de armazenamento de água disponível do solo.

Os incrementos na produtividade de colmos superiores a $140 \%$, sob irrigação plena, podem ser atribuídos à disponibilidade hídrica adequada durante todo o período de crescimento, o que proporciona maior disponibilidade de nutrientes para o sistema radicular da cana-de-açúcar. Consequentemente, ocorre remobilização de energia e de carboidratos das raízes, para aumento do perfilhamento e alongamento do colmo (Inman-Bamber \& Smith, 2005; Singh et al., 2007), que é estimulado pela produção do hormônio vegetal citoquinina, responsável pelo crescimento vegetativo da parte aérea das plantas (Takei et al., 2002).

Quando avaliadas separadamente entre os sistemas de produção irrigado e sequeiro, as variedades de cana-de-açúcar apresentaram diferentes comportamentos para produtividade de colmos (Tabela 2). Sob irrigação, as variedades RB92579, SP81-3250 e RB763710 foram mais produtivas, com acréscimos de $73,6,50,5$ e 29,9 $\mathrm{Mg} \mathrm{ha}^{-1}$, respectivamente. As demais variedades não diferiram entre si, tendo apresentado produtividade média de $182 \mathrm{Mg} \mathrm{ha}^{-1}$. Não foram observadas diferenças significativas entre os resultados de produtividade de colmos das variedades conduzidas sem irrigação.

Para produtividade de açúcar, houve diferença entre os sistemas de produção, com ganhos de $18 \mathrm{Mg} \mathrm{ha}^{-1}$, sob irrigação plena (Tabela 2). Entre as variedades, a RB943365 e a RB92579 apresentaram incrementos de $215 \%$, em comparação às condições de sequeiro. $\mathrm{O}$ menor incremento foi observado para a variedade RB813804, enquanto as demais variedades obtiveram ganhos médios de $140 \%$. O aumento na produtividade de açúcar com o uso da irrigação também foi observado em outros estudos que avaliaram o efeito de diferentes lâminas de irrigação, em comparação ao cultivo de sequeiro (Wiedenfeld, 2000; Moura et al., 2005; Dantas Neto et al., 2006; Carvalho et al., 2009). No entanto, os incrementos obtidos não ultrapassaram $60 \%$, com uso da irrigação.
Entre as variedades irrigadas, a RB92579 e a SP81-3250 foram as mais produtivas em termos de açúcar, tendo apresentado incrementos de 15,3 e $10,1 \mathrm{Mg} \mathrm{ha}^{-1}$, respectivamente, em comparação às variedades RB72454, SP79-1011, RB763710, RB813804, RB863129 e SP78-4764, que se mostraram menos produtivas, com valor médio de $27,2 \mathrm{Mg} \mathrm{ha}^{-1}$. As variedades RB943365, RB872552 e RB867515 apresentaram produtividade média de açúcar de 31,6 $\mathrm{Mg} \mathrm{ha}^{-1}$, superior ao observado em canaviais irrigados no Brasil (Moura et al., 2005; Dantas Neto et al., 2006; Carvalho et al., 2009) e nos Estados Unidos (Wiedenfeld, 1995, 2000; Wiedenfeld \& Enciso, 2008). No regime de sequeiro, as médias de produtividade de açúcar não diferiram significativamente entre as variedades (Tabela 2).

$\mathrm{O}$ uso da irrigação proporcionou maior EUA disponível, com produção de, em média, $70,2 \mathrm{~kg} \mathrm{ha}^{-1} \mathrm{~mm}^{-1}$ de colmos a mais, em comparação às variedades conduzidas em regime de sequeiro (Tabela 2). O aumento na EUA com uso da irrigação também foi constatado por Singh \& Mohan (1994) e Farias et al. (2008b), em diferentes condições edafoclimáticas. Segundo Farias et al. (2008a), a cana-de-açúcar irrigada emite mais rapidamente o sistema radicular e permanece com um volume maior de raiz na superfície, em comparação ao regime de sequeiro. Portanto, após irrigações ou chuvas, há maior absorção de água e nutrientes (Lampurlanés et al., 2001), o que se reflete diretamente no aumento da produção de colmos e na maior EUA (Singh et al., 2007).

Quando irrigada plenamente, a variedade RB92579 mostrou-se a mais eficiente na utilização da água absorvida, seguida das variedades SP81-3250 e RB763710 (Tabela 2). As demais variedades não diferiram significativamente quanto à EUA, com valores médios de $130 \mathrm{~kg} \mathrm{ha}^{-1} \mathrm{~mm}^{-1}$. Para as variedades conduzidas sem irrigação, a EUA não apresentou diferença significativa.

Com relação aos atributos tecnológicos, houve diferença apenas para ${ }^{\circ}$ Brix, Pol e fibra, entre os regimes hídricos, que apresentaram decréscimo de 1,1, 0,7 e 2,5\% com uso da irrigação, respectivamente (Tabela 3 ). Esses resultados estão de acordo com os obtidos por Deon et al. (2010), que verificaram redução significativa para ${ }^{\circ}$ Brix, sob irrigação, mas não observaram diferença para ATR e PC. No entanto, os autores não constataram 
diferenças entre os regimes hídricos para a variável Pol, como verificado no presente trabalho. Dalri \& Cruz (2008) também obtiveram baixos valores para ${ }^{\circ}$ Brix e Pol, em cana irrigada, em Nitossolo Vermelho, nas condições edafoclimáticas de Botucatu, SP.

Entre as variedades, os atributos ${ }^{\circ} \mathrm{Brix}$, Pol, ATR e PC diferiram significativamente apenas em RB72454 e RB763710 quando irrigadas, com redução média de 3,6\%, 3,4\%, 21,8 $\mathrm{kg} \mathrm{Mg}^{-1}$ e 2,6\%, em comparação ao regime de sequeiro (Tabela 3 ). Para as demais variedades, a concentração de açúcar no caldo e no colmo não diferiu das cultivadas sem irrigação, exceto para RB943365 quando irrigada, que apresentou ganhos em Pol, ATR e $\mathrm{PC}$ de 2,6\%, 33,2 $\mathrm{kg} \mathrm{Mg}^{-1}$ e 4,2\%, respectivamente.

Os valores elevados dos atributos tecnológicos das variedades irrigadas, com 12 meses de desenvolvimento, podem estar associados ao manejo pleno da irrigação, que intensifica o perfilhamento e a elongação do colmo e, consequentemente, antecipa a maturação fisiológica da cana-de-açúcar e promove o aumento dos níveis de sacarose nas células do colmo. Tognetti et al. (2003) obtiveram resultados semelhantes, ao verificar aceleração da maturação e antecipação do período de colheita com uso da irrigação.
Quando irrigadas, as variedades RB72454 e RB763710 apresentaram menor valor de ${ }^{\circ}$ Brix, Pol, ATR e PC. No regime de sequeiro, foram observadas diferenças apenas para as variedades RB813804, RB72454, SP78-4764 e RB867515, com maiores ${ }^{\circ}$ Brix e Pol, e para RB943365, com menor ATR e PC.

Nas variedades conduzidas sob irrigação plena, a idade fisiológica de 12 meses proporcionou teores de fibra menores em comparação às variedades conduzidas sem irrigação, com exceção da variedade RB943365, que não apresentou diferença entre os regimes hídricos adotados. No cultivo de sequeiro, o teor de fibra não diferiu entre as variedades. Entretanto, os valores médios de 12\%, encontrados para as variedades RB813804, RB72454 e RB763710 quando irrigadas, foram inferiores à média de $13,3 \%$, obtida pelas demais variedades manejadas sob o mesmo sistema de produção.

Como o teor de fibra aumenta a resistência à extração do caldo, recomenda-se valor médio entre 10,5 e $12,5 \%$ para a manutenção energética das indústrias que processam a cana-de-açúcar (Oliveira et al., 2009). Portanto, o teor médio da fibra, de $12,9 \%$, observado para as variedades irrigadas pode ser considerado adequado e não interfe na eficiência da extração do caldo. Contudo,

Tabela 3. Atributos tecnológicos de diferentes variedades de cana-de-açúcar cultivadas em regime hídrico de sequeiro e sob irrigação plena, de 2006 a $2007^{(1)}$.

\begin{tabular}{|c|c|c|c|c|c|c|c|c|c|c|}
\hline \multirow[t]{3}{*}{ Variedade } & \multicolumn{4}{|c|}{ Atributos do caldo } & \multicolumn{6}{|c|}{ Atributos do colmo } \\
\hline & \multicolumn{2}{|c|}{${ }^{\circ}$ Brix $(\%)$} & \multicolumn{2}{|c|}{ Pol (\%) } & \multicolumn{2}{|c|}{$\operatorname{ATR}\left(\mathrm{kg} \mathrm{Mg}^{-1}\right)$} & \multicolumn{2}{|c|}{ Sacarose no colmo $(\%)$} & \multicolumn{2}{|c|}{ Teor de fibra $(\%)$} \\
\hline & Sequeiro & Irrigado & Sequeiro & Irrigado & Sequeiro & Irrigado & Sequeiro & Irrigado & Sequeiro & Irrigado \\
\hline & \multicolumn{10}{|c|}{ Variedades de maturação precoce } \\
\hline SP79-1011 & $21,0 \mathrm{bA}$ & $20,6 \mathrm{aA}$ & $18,3 \mathrm{bA}$ & $18,5 \mathrm{aA}$ & $144,7 \mathrm{aA}$ & $157,6 \mathrm{aA}$ & $15,0 \mathrm{aA}$ & $16,6 \mathrm{aA}$ & $16,1 \mathrm{aA}$ & $13,2 \mathrm{aB}$ \\
\hline RB813804 & $22,5 \mathrm{aA}$ & $20,0 \mathrm{aA}$ & $20,3 \mathrm{aA}$ & $17,7 \mathrm{aA}$ & $164,1 \mathrm{aA}$ & $152,8 \mathrm{aA}$ & $17,3 \mathrm{aA}$ & $15,9 \mathrm{aA}$ & $16,0 \mathrm{aA}$ & $12,0 \mathrm{bB}$ \\
\hline RB863129 & $20,6 \mathrm{bA}$ & $20,1 \mathrm{aA}$ & $17,9 \mathrm{bA}$ & $17,8 \mathrm{aA}$ & $145,7 \mathrm{aA}$ & $150,7 \mathrm{aA}$ & $15,0 \mathrm{aA}$ & $16,0 \mathrm{aA}$ & $14,6 \mathrm{aA}$ & $13,1 \mathrm{aB}$ \\
\hline RB872552 & $21,0 \mathrm{bA}$ & $20,1 \mathrm{aA}$ & $18,3 \mathrm{bA}$ & $18,3 \mathrm{aA}$ & $151,4 \mathrm{aA}$ & $156,0 \mathrm{aA}$ & $15,9 \mathrm{aA}$ & $16,4 \mathrm{aA}$ & $16,2 \mathrm{aA}$ & $13,1 \mathrm{aB}$ \\
\hline \multirow[t]{2}{*}{ RB943365 } & $19,9 \mathrm{bA}$ & $20,8 \mathrm{aA}$ & $16,4 \mathrm{bB}$ & $19,0 \mathrm{aA}$ & $130,6 \mathrm{bB}$ & $163,8 \mathrm{aA}$ & $13,1 \mathrm{bB}$ & $17,3 \mathrm{aA}$ & $15,1 \mathrm{aA}$ & $14,1 \mathrm{aA}$ \\
\hline & \multicolumn{10}{|c|}{ Variedades de maturação média à tardia } \\
\hline RB72454 & $22,0 \mathrm{aA}$ & $17,4 \mathrm{bB}$ & $19,2 \mathrm{aA}$ & $14,8 \mathrm{bB}$ & $155,0 \mathrm{aA}$ & $124,8 \mathrm{bB}$ & $16,1 \mathrm{aA}$ & $12,6 \mathrm{bB}$ & $15,3 \mathrm{aA}$ & $12,4 \mathrm{bB}$ \\
\hline RB763710 & $20,4 \mathrm{bA}$ & $17,8 \mathrm{bB}$ & $17,5 \mathrm{bA}$ & $15,1 \mathrm{bB}$ & $142,9 \mathrm{aA}$ & $129,5 \mathrm{bB}$ & $14,8 \mathrm{aA}$ & $13,0 \mathrm{bB}$ & $15,2 \mathrm{aA}$ & $11,7 \mathrm{bB}$ \\
\hline SP78-4764 & $21,5 \mathrm{aA}$ & $23,0 \mathrm{aA}$ & $18,8 \mathrm{aA}$ & $18,2 \mathrm{aA}$ & $149,7 \mathrm{aA}$ & $152,8 \mathrm{aA}$ & $15,6 \mathrm{aA}$ & $16,0 \mathrm{aA}$ & $15,9 \mathrm{aA}$ & $13,6 \mathrm{aB}$ \\
\hline SP81-3250 & $20,5 \mathrm{bA}$ & $20,6 \mathrm{aA}$ & $18,1 \mathrm{bA}$ & $18,1 \mathrm{aA}$ & $148,6 \mathrm{aA}$ & $152,8 \mathrm{aA}$ & $15,5 \mathrm{aA}$ & $16,2 \mathrm{aA}$ & $14,5 \mathrm{aA}$ & $12,9 \mathrm{aB}$ \\
\hline RB867515 & $21,4 \mathrm{aA}$ & $20,7 \mathrm{aA}$ & $18,8 \mathrm{aA}$ & $18,2 \mathrm{aA}$ & $151,1 \mathrm{aA}$ & $153,6 \mathrm{aA}$ & $15,7 \mathrm{aA}$ & $16,0 \mathrm{aA}$ & $15,5 \mathrm{aA}$ & $13,0 \mathrm{aB}$ \\
\hline RB92579 & $21,2 \mathrm{bA}$ & $21,1 \mathrm{aA}$ & $18,2 \mathrm{bA}$ & $18,8 \mathrm{aA}$ & $146,8 \mathrm{aA}$ & $158,9 \mathrm{aA}$ & $15,1 \mathrm{aA}$ & $16,6 \mathrm{aA}$ & $15,0 \mathrm{aA}$ & $13,1 \mathrm{aB}$ \\
\hline Média & $21,1 \mathrm{~A}$ & $20,0 \mathrm{~B}$ & $18,4 \mathrm{~A}$ & $17,7 \mathrm{~B}$ & 148,2 & 150,3 & 15,4 & 15,7 & $15,4 \mathrm{~A}$ & $12,9 \mathrm{~B}$ \\
\hline CV (\%) & \multicolumn{2}{|c|}{3,82} & \multicolumn{2}{|c|}{5,42} & \multicolumn{2}{|c|}{6,15} & \multicolumn{2}{|c|}{7,56} & \multicolumn{2}{|c|}{6,36} \\
\hline Variedade (Var) & \multicolumn{2}{|c|}{$5,38^{* *}$} & \multicolumn{2}{|c|}{$5,14 * *$} & \multicolumn{2}{|c|}{$3,79^{* *}$} & \multicolumn{2}{|c|}{$5,53 * *$} & \multicolumn{2}{|c|}{$1,96 * *$} \\
\hline Regime hídrico (Rh) & \multicolumn{2}{|c|}{$41,18^{*}$} & \multicolumn{2}{|c|}{$11,65 * *$} & \multicolumn{2}{|c|}{$1,12 \mathrm{~ns}$} & \multicolumn{2}{|c|}{$1,72 \mathrm{~ns}$} & \multicolumn{2}{|c|}{$166,59 * *$} \\
\hline Var $x \mathrm{Rh}$ & \multicolumn{2}{|c|}{$7,78 * *$} & \multicolumn{2}{|c|}{$7,21 * *$} & \multicolumn{2}{|c|}{$6,34 * *$} & \multicolumn{2}{|c|}{$5,91 * *$} & \multicolumn{2}{|c|}{$2,05 * *$} \\
\hline
\end{tabular}

${ }^{(1)}$ Médias seguidas de letras iguais, maiúsculas nas linhas e minúsculas nas colunas, não diferem entre si pelo teste de Scott-Knott, a 5 \% de probabilidade. ns, Não significativo; ** e *, Significativo pelo teste F, a 1 e 5\% e de probabilidade, respectivamente. Pol, sacarose aparente no caldo; ATR, Açúcar teórico recuperável. 
o resultado obtido para as variedades manejadas sem irrigação $(15,4 \%)$, com 17 meses de crescimento, foi superior à média recomendada pelos autores.

$\mathrm{O}$ uso da irrigação promoveu as maiores produtividades de colmos e de açúcar, a maior eficiência de uso da água e a concentração adequada de açúcar com menores valores de fibra, o que indica a viabilidade técnica deste manejo como alternativa para o aumento da produtividade dos canaviais brasileiros.

\section{Conclusões}

1. A irrigação plena proporciona maiores ganhos médios na produtividade de colmo e de açúcar, e maior eficiência de uso da água absorvida.

2. As variedades de cana-de-açúcar RB92579 e SP81-3250 de maturação média à tardia se destacam quanto à produtividade de colmos e de açúcar e à eficiência de uso da água, e são recomendas para estudos de resposta à irrigação pela cana-de-açúcar.

3.É possível manejar as variedades de cana-de-açúcar de maturação precoce e de média à tardia em áreas irrigadas durante o período de colheita, para minimizar as variações na qualidade e na produção de açúcar.

\section{Agradecimentos}

Ao Conselho Nacional de Desenvolvimento Científico e Tecnológico, pelo apoio financeiro; e à Estação experimental de Cana-de-açúcar de Carpina, pelo apoio na instalação e na condução do experimento.

\section{Referências}

CARVALHO, C.M. de; AZEVEDO, H.M. de; DANTAS NETO, J.; FARIAS, C.H. de A.; SILVA, C.T.S. da; GOMES FILHO, R.R. Rendimento de açúcar e álcool da cana-de-açúcar submetida a diferentes níveis de irrigação. Revista Brasileira de Ciências Agrárias, v.4, p.72-77, 2009.

CARVALHO, C.M. de; AZEVEDO, H.M. de; DANTAS NETO, J.; MELO, E.P. de; SILVA, C.T.S. da; GOMES FILHO, R.R. Resposta dos parâmetros tecnológicos da terceira folha de cana-de-açúcar submetida a diferentes níveis de irrigação. Revista Brasileira de Ciências Agrárias, v.3, p.337-342, 2008.

DALRI, A.B.; CRUZ, R.L. Produtividade da cana-de-açúcar fertirrigada com $\mathrm{N}$ e K via gotejamento subsuperficial. Engenharia Agrícola, v.28, p.516-524, 2008.

DANTAS NETO, J.; FIGUEREDO J.L. da C.; FARIAS, C.H. de A.; AZEVEDO, H.M.; AZEVEDO, C.A.V. de. Resposta da cana-de-açúcar, primeira soca, a níveis de irrigação e adubação de cobertura. Revista Brasileira de Engenharia Agrícola e Ambiental, v.10, p.283-288, 2006.
DEON, M.D.; GOMES, T.M.; MELFI, A.J.; MONTES, C.R.; SILVA, E. da. Produtividade e qualidade da cana-de-açúcar irrigada com efluente de estação de tratamento de esgoto. Pesquisa Agropecuária Brasileira, v.45, p.1149-1156, 2010.

DOORENBOS, J.; DRUITT, W.O. Las necessidades de agua de los cultivos. Roma: FAO, 1974. 194p. (Estudio FAO, Riego y Drenaje, 24).

DOORENBOS, J.; KASSAM, A.K. Efeito da água no rendimento das culturas. Campina Grande: Universidade Federal da Paraíba, 1994. 306p.

EFEOGLU, B.; EKMEKÇI, Y.; ÇIÇEK, N. Physiological responses of three maize cultivars to drought stress and recovery. South African Journal of Botany, v.75, p.34-42, 2009.

FARIAS, C.H. de A.; FERNANDES, P.D.; AZEVEDO, H.M.; DANTAS NETO, J. Índices de crescimento da cana-de-açúcar irrigada e de sequeiro no Estado da Paraíba. Revista Brasileira de Engenharia Agrícola e Ambiental, v.12, p.356-362, 2008a.

FARIAS, C.H. de A.; FERNANDES, P.D.; DANTAS NETO, J.; GHEYI, H.R. Eficiência no uso da água na cana-de-açúcar sob diferentes lâminas de irrigação e níveis de zinco no litoral paraibano. Engenharia Agrícola, v.28, p.494-506, 2008b.

FERNANDES, A.C. Cálculos na agroindústria da cana-de-açúcar. 2.ed. Piracicaba: STAB, 2003. 240p.

GASCHO, G.J. Water-sugarcane relationships. Sugar Journal, v.48, p.11-17, 1985.

INMAN-BAMBER, N.G. Sugarcane water stress criteria for irrigation and drying off. Field Crops Research, v.89, p.107-122, 2004.

INMAN-BAMBER, N.G.; SMITH, D.M. Water relations in sugarcane and response to water deficits. Field Crops Research, v.92, p.185-202, 2005.

LAMPURLANÉS, J.; ANGÁS, J.; CANTERO-MARTINEZ, C. Root growth, soil water content and yield of barley under different tillage systems on two soils in semiarid conditions. Field Crop Research, v.69, p.27-40, 2001.

MACHADO, R.S.; RIBEIRO, R.V.; MARCHIORI, P.E.R.; MACHADO, D.F.S.P.; MACHADO, E.C.; LANDELL, M.G. de A. Respostas biométricas e fisiológicas ao déficit hídrico em cana-de-açúcar em diferentes fases fenológicas. Pesquisa Agropecuária Brasileira, v.44, p.1575-1582, 2009.

MOURA, M.V.P. da S.; FARIAS, C.H. de A.; AZEVEDO, C.A.V. de; DANTAS NETO, J.; AZEVEDO, H.M. de; PORDEUS, R.V. Doses de adubação nitrogenada e potássica em cobertura na cultura da cana-de-açúcar, primeira soca, com e sem irrigação. Ciência Agrotécnica, v.29, p.753-760, 2005.

OLIVEIRA, E.C.A. de; OLIVEIRA, R.I. de; ANDRADE, B.M.T. de; FREIRE, F.J.; LIRA JÚNIOR, M.A.; MACHADO, P.R. Crescimento e acúmulo de matéria seca em variedades de cana-de-açúcar cultivadas sob irrigação plena. Revista Brasileira de Engenharia Agrícola e Ambiental, v.14, p,951-960, 2010.

OLIVEIRA, E.L. de; ANDRADE, L.A. de B.; FARIA, M.A. de; EVANGELISTA, A.W.P.; MORAIS, A.R. de. Uso da vinhaça de alambique e nitrogênio em cana-de-açúcar irrigada e não irrigada. Pesquisa Agropecuária Brasileira, v.44, p.1398-1403, 2009. 
RAIZER, A.J.; VENCOVSKY, R. Estabilidade fenotípica de novas variedades de cana-de-açúcar para o estado de São Paulo. Pesquisa Agropecuária Brasileira, v.34, p.2241-2246, 1999.

ROBERTSON, M.J.; INMAN-BAMBER, N.G.; MUCHOW, R.C.; WOOD, A.W. Physiology and productivity of sugarcane with early and mid-season water deficit. Field Crops Research, v.64, p.211-227, 1999.

SANTOS, H.G. dos; JACOMINE, P.K.T.; ANJOS, L.H.C. dos; OLIVEIRA, V.A. de; OLIVEIRA, J.B. de; COELHO, M.R.; LUMBRERAS, J.F.; CUNHA, T.J.F. (Ed.). Sistema brasileiro de classificação de solos. 2.ed. Rio de Janeiro: Embrapa Solos, 2006. $306 \mathrm{p}$.

SAS INSTITUTE. SAS/STAT user's guide: statistics. Version 8. Cary: SAS Institute, 1999.

SILVA, A.L.C. da; COSTA, W.A.J.M. de. Varietal variation in growth, physiology and yield of sugarcane under two contrasting water regimes. Tropical Agricultural Research, v.16, p.1-12, 2004.

SINGH, P.N.; MOHAN, S.C. Water use and yield response of sugarcane under different irrigation schedules and nitrogen levels in a subtropical region. Agricultural Water Management, v.26, p.253-264, 1994.

SINGH, P.N.; SHUKLA, S.K.; BHATNAGAR, V.K. Optimizing soil moisture regime to increase water use efficiency of sugarcane (Saccharum spp. Hybrid complex) in subtropical
India. Agricultural Water Management, v.90, p.95-100, 2007.

SOUZA, E.F.; BERNADO, S.; CARVALHO, J.A. Função de produção da cana-de-açúcar em relação à água para três variedades em Campos dos Goytacazes. Engenharia Agrícola, v.19, p.28-12, 1999.

TAKEI, K.; TAKAHASHI, T.; SUGIYAMA, T.; YAMAYA, T.; SAKAKIBARA, H. Multiple routes communicating nitrogen availability from roots to shoots: a signal transduction pathway mediated by cytokinin. Journal of Experimental Botany, v.53, p.971-977, 2002.

TOGNETTI, R.; PALLADINO, M.; MINNOCCI, A.; DELFINE, D.; ALVINO, A. The response of sugar beet to drip and low-pressure sprinkler irrigation in southern Italy. Agricultural Water Management, v.60, p.135-155, 2003.

WIEDENFELD, R.P. Effects of irrigation and $\mathrm{N}$ fertilizer application on sugarcane yield and quality. Field Crops Research, v.43, p.101-108, 1995.

WIEDENFELD, R.P. Water stress during different sugarcane growth periods on yield and response to $\mathrm{N}$ fertilization. Agricultural Water Management, v.43, p.173-182, 2000.

WIEDENFELD, B.; ENCISO, J. Sugarcane responses to irrigation and nitrogen in semiarid south Texas. Agronomy Journal, v.100, p.665-671, 2008 .

ZHENG, S.J.; YANG, J.L. Target sites of aluminum phytotoxicity. Biologia Plantarum, v.49, p.321-331, 2005.

Recebido em 12 de março de 2011 e aprovado em 5 de maio de 2011 